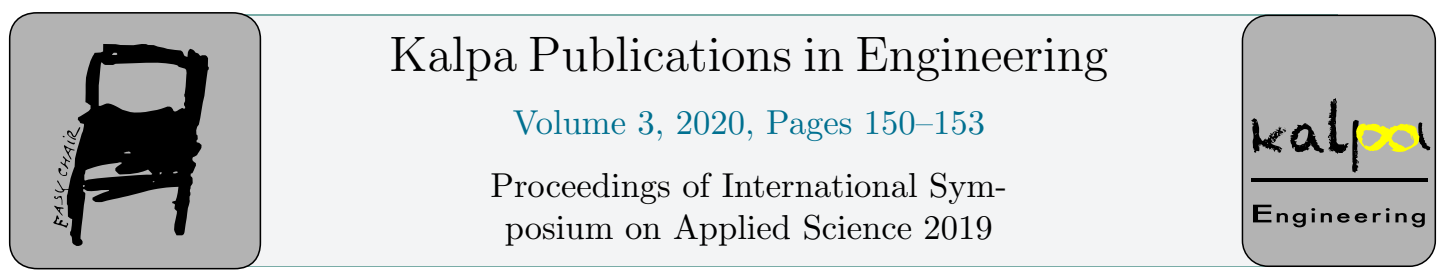

\title{
Pulse Generation Behavior of Single-Walled Carbon Nanotube/ Polyoxometalate Complex Random Network
}

\author{
Deep Banerjee ${ }^{1}$, Yu Xinguang ${ }^{1}$, Detiza Goldianto Octensi Hernowo ${ }^{1}$, \\ Hirofumi Tanaka ${ }^{1}$, Yoshito Yamazaki ${ }^{2}$, Takuji Ogawa ${ }^{2}$ \\ ${ }^{1}$ Kyushu Institute of Technology \\ ${ }^{2}$ Osaka University \\ dban89@gmail.com
}

\begin{abstract}
Replacing von-Neumann architecture with walled carbon nanotube (SWNT) with new neuromorphic chips is vital for computers to perform polyoxometalate POM) ion ([SV2W10O40 ]H4TPP) complex cognitive problem with low power and see the effect of higher POM concentration. We consumption like our brain. The synaptic part of the use simple sonication method for SWNT/POM brain has been well studied via memristors but fabrication and study the spiking and spike time research on artificial neuron is only limited to high interval behavior via electrical measurements. The power CMOS circuitry. Following the previous work results show that the device can behave as an artificial by $\mathrm{H}$. Tanaka et al.[2] herein we functionalize single neuron even at low voltages and hence can be used for low power neuromorphic computing.
\end{abstract}

\section{Introduction}

The advent of artificial intelligence has paved way to the development of more energy efficient, fault tolerant and fast processing brain like computers. Recent studies have shown that adequate noise facilitates information acquisition and processing in biological systems [1]. To exploit this phenomenon carbon nanotubes functionalized with redox active molecules has proven to be a good candidate for generating large electrical noise with rich dynamics [2] but at a very large voltage of 150V. Here we report a different POM molecule adsorbed on the sidewall of SWNT and study the spiking behavior with the change in POM moiety and POM loading onto the SWNT. 
Pulse Generation Behavior of Single-Walled Carbon Nanotube/Polyoxometalate CRN Banerjee et al.

\section{Materials and Method}

The HiPCO SWNT was as purchased and was purified using usual acid reflux method. The POM ([SV2W10O40]H4TPP) was obtained from Osaka University and was as used. The SWNT/POM was synthesized by sonication method [3] in ethanol solvent. Two different loading of POM, $0.25 \mathrm{wt} \%$ and $20 \mathrm{wt} \%$ relative to SWNT were fabricated. The resultant dispersion was vacuum filtered and the film was transferred onto a glass substrate using acetone vapor assisted method. Al electrodes were sputtered to carry out electrical measurements. DAQ system operated by LabView combined with a function generator was used for electric studies.

\section{Results and Discussion}

The POM has four porphyrin moieties (Figure 1) which binds to the SWNT via $\pi$ - $\pi$ interaction. From Figure 2(a) and 2(b) we can see that the SWNT/POM network exhibits negative spike due the presence of porphyrin ring which is electron donating thereby inducing hole current to SWNT. Higher POM loading allows more molecules to be adsorbed onto the SWNT surface leading to increased capacitance and more electron transfer from the counteraction thus generating negative spike at low voltage spike compared to low POM loading. The complex also shows a positive spike behavior at high voltage of $20 \mathrm{~V}$ as seen from Figure 3 which indicates that the POM molecule accts as an electron sponge [4] and undergoes multiple redox processes at different voltages. The spike timing interval was studied by plotting all $(\mathrm{tn}, \mathrm{tn}+1)$ interval between the $(\mathrm{n}-1)$ th, and $(\mathrm{n}+1)$ th pulse called a Poincare plot[5] as shown in Figure 4 which reveals where the spike intervals for $2.5 \mathrm{~V}$ are mostly centered on $1 \mathrm{~s}$, whereas the one from $15 \mathrm{~V}$ and $20 \mathrm{~V}$ are shifted towards higher time interval. Such shift of spike time interval with increase in voltage is still unclear at this point and further investigation is required for a firm explanation.

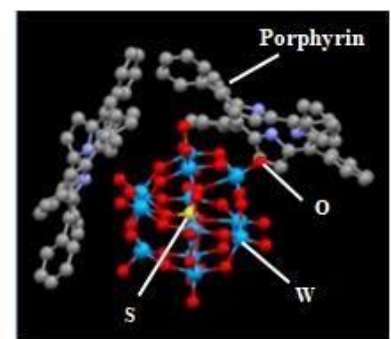

Figure 1: Structure of [ $\left.\mathrm{SV}_{2} \mathrm{~W}_{10} \mathrm{O}_{40}\right]$ H4TPP Four porphyrin rings surround POM

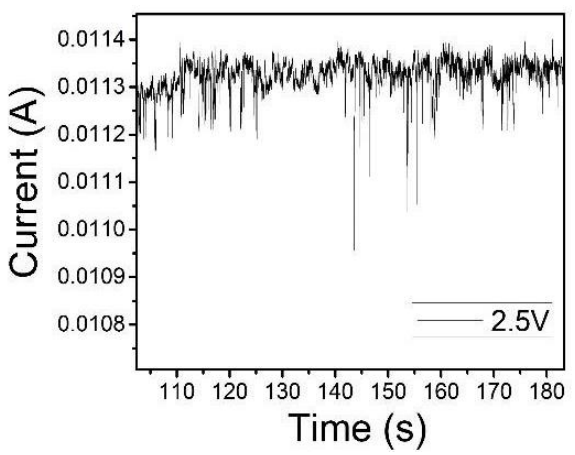

(b)

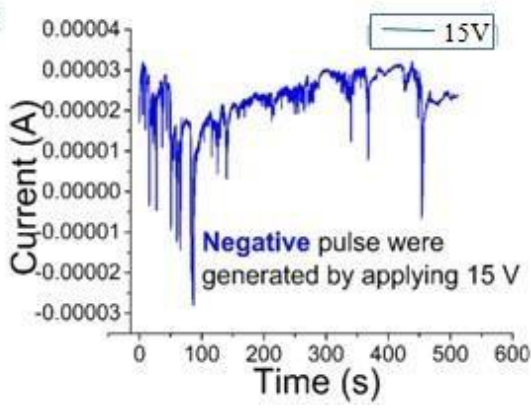

Figure 2: Negative pulse generation in (a) $\mathrm{SWNT} /\left[\mathrm{SV}_{2} \mathrm{~W}_{10} \mathrm{O}_{40}\right] \mathrm{H} 4 \mathrm{TPP}$ at $2.5 \mathrm{~V}$ (SWNT:POM = 1:20), (b) $\mathrm{SWNT} /\left[\mathrm{SV}_{2} \mathrm{~W}_{10} \mathrm{O}_{40}\right] \mathrm{H}_{4}$ at $15 \mathrm{~V}(\mathrm{SWNT}: \mathrm{POM}=1: 0.25)$ 
Pulse Generation Behavior of Single-Walled Carbon Nanotube/Polyoxometalate CRN Banerjee et al.

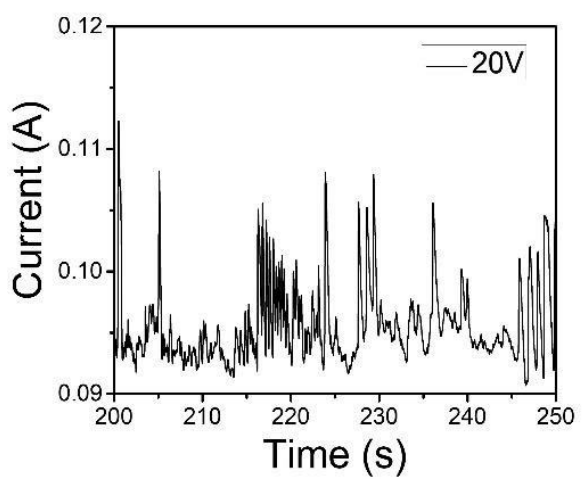

Figure 3: Positive pulse generation in $\mathrm{SWNT} /\left[\mathrm{SV}_{2} \mathrm{~W}_{10} \mathrm{O}_{40}\right] \mathrm{H}_{4} \mathrm{TPP}$ at $20 \mathrm{~V}(\mathrm{SWNT}: \mathrm{POM}=1: 20)$.

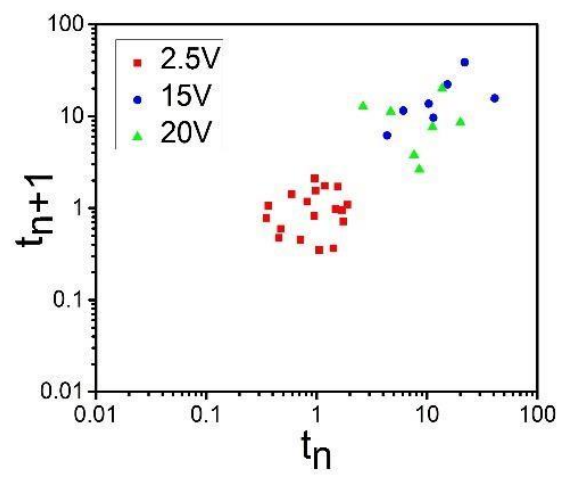

Figure 4: Return map analysis using Poincare plot obtained from I-t measurement.

\section{Conclusion}

We have successfully demonstrated an artificial spiking neuron by using SWNT/POM. The charge storing ability of POM allows it to undergo multiple charge-discharge processes which ultimately results in such spike signal generation. Although a negative spiking tendency was observed for a POM with a porphyrin moiety compared to positive spikes in previous report [2], more studies on the POM to SWNT loading might lead us to better optimized results both in terms of spike generation over a large potential window and spiking time interval. Such a complex semi-conducting which configures to a wide range of voltages and with neuronal spike like behavior can be implemented as a neuromorphic hardware device in order to solve complex cognitive problems of image, pattern and speech recognition in near future. 
Pulse Generation Behavior of Single-Walled Carbon Nanotube/Polyoxometalate CRN Banerjee et al.

\section{References}

T. Aihara et al., How does stochastic resonance work within the human brain? - Psychophysics of internal and external noise. arXiv preprint arXiv: 1804.08150 (2018).

H. Tanaka et al., A molecular neuromorphic network device consisting of single-walled carbon nanotubes complexed with polyoxometalate. Nat. Commun. 9, 2693 (2018)

$\mathrm{J}$. Hu et al. Wiring redox-active polyoxometalates to carbon nanotubes using a sonication-driven periodic functionalization strategy. Energy Environ. Sci. 9, 1095-1101 (2016)

$\mathrm{H}$. Wang et al. In operando X-ray absorption fine structure studies of polyoxometalate molecular cluster batteries: polyoxometalates as electron sponges. J. Am. Chem. Soc. 134, 4918-4924 (2012)

H. V. Huikuri et al. Fractal correlation properties of R-R interval dynamics and mortality in patients with depressed left ventricular function after an acute myocardial infarction. Circulation 101, 47-53 (2000) 